\title{
Atrial fibrillation surgery and mitral repair
}

\author{
Manuel Castellá \\ Department of Cardiovascular Surgery, Cardiovascular Institute, Hospital Clínic, University of Barcelona, Barcelona, Spain \\ Correspondence to: Manuel Castellá, MD, PhD, MBA. Department of Cardiovascular Surgery, Cardiovascular Institute, Hospital Clínic, Villarroel 170, \\ 08036 Barcelona, Spain. Email: mcaste@clinic.ub.es.
}

Received: 16 July 2017; Accepted: 22 August 2017.

doi: 10.21037/jovs.2017.08.22

View this article at: http://dx.doi.org/10.21037/jovs.2017.08.22

\section{Introduction}

Mitral disease is known to be associated to atrial fibrillation (AF) for decades. The most common association is with mitral stenosis, which produces an important dilatation and fibrosis of the left atrium due to volume overload. Interestingly, in young patients with mitral disease, sinus rhythm is maintained even with a very enlarged left atrium, but when AF starts, it is to stay in what is was called chronic AF and nowadays persistent and after one year, long-standing AF. Additionally, AF in these patients tends to be of low voltage with a controlled frequency, usually lower than $100-110 \mathrm{bpm}$. This is totally opposite to the paroxysmal AF episodes of patients with no mitral disease, in which electrical triggers within the pulmonary veins may be the most probable cause to start this arrhythmia. These later patients usually have more symptoms related to a faster AF. Over the years, paroxysmal AF can transform to persistent and later on long-standing persistent $\mathrm{AF}$, with progressive dilatation of the left atrium.

Mitral regurgitation also may dilate and fibrose the left atrium due to pressure and volume overload similar to mitral stenosis, although usually to a lower stand. Up to now it has been assumed that the evidence for AF physiopathology and treatment is similar for both mitral stenosis and regurgitation.

\section{Why to treat AF in mitral insufficiency}

Due to the previous statements about the physiopathology of AF in patients with mitral disease, AF treatment by catheter ablation without addressing valve insufficiency has poor results. Indeed, all major guidelines and expert consensus statements such as the 2016 ESC/EACTS Guidelines for Atrial Fibrillation Management do not recommend catheter ablation in patients with severe mitral regurgitation (1). On the contrary, these Guidelines state that mitral repair and Maze procedure should be considered in asymptomatic patients with mitral regurgitation on the new onset of AF, even if there is no ventricular dilatation or pulmonary hypertension, when repair is feasible. This is supported by studies that have described AF as one of the three factors influencing late survival after mitral repair, together with age and left ventricular dysfunction (2).

Concomitant AF surgery has demonstrated to be a safe and effective procedure. The 2016 ESC/EACTS guidelines commissioned a systematic review of the literature to the Cochrane Collaboration Organization to evaluate all evidence around concomitant AF surgery (3). This review of over 21 trials and 31 reports concluded that AF surgery significantly increased freedom from $\mathrm{AF}$ and other atrial arrhythmias compared to non-AF surgery. Additionally, by adding AF surgery there was no significant difference in all-cause mortality, 30-day mortality, cardiovascular related mortality, adverse events as defined by the investigators, neurologic or thromboembolic events or need for surgical re-exploration. The only statistically significant complication associated to add AF ablation to a cardiac procedure was an increased rate of pacemaker implantation from $4.3 \%$ to $6.4 \%$ [RR 1.69; 95\% CI: $1.12-2.54$; $n=1,631$ from 17 randomized control trials (RCTs)]. Interestingly, from the 17 RCTs analyzed, only one had a significant increase of pacemaker implantation, with a rate of $19.5 \%$ in the concomitant AF surgery group (4).

While preoperative $\mathrm{AF}$ is known to influence late stroke and death after mitral surgery, and concomitant AF surgery is known to significantly reduce atrial arrhythmias, it is been very difficult to demonstrate clinical benefits of surgical ablation in terms of mortality or stroke. No randomized 
controlled trial has shown clear clinical improvements and only some personal series have suggested benefits in stroke or survival (5-8). But very recent data analysis from the STS database matching 28,739 patient-pairs with and without AF described that surgical ablation was associated with a reduction in mortality and stroke, while an increase in renal failure and the need for pacemaker implantation (9). Most probably, surgical ablation for AF really reduces long-term mortality and risk for stroke, but to demonstrate this benefit a big population with a long follow-up is needed. Up to now all randomized controlled trials in AF surgery had one or two years follow-up, with no more than 270 patients, and only personal series have reached 10 years follow-up.

\section{Factors influencing outcomes}

Several factors influence results in concomitant AF surgery. Probably the most important is left atrial dilatation, which is associated to muscular deterioration and fibrosis. Several studies have indicated that results of AF surgery in patients with left atrium beyond $60 \mathrm{~mm}$ diameter drop below $50 \%$ at one year (10). Other factors influencing outcomes are age, AF duration, type of AF and surgeon's experience (11). Type of AF deserves a special comment. Most patients with $\mathrm{AF}$ and mitral regurgitation have persistent or longstanding persistent AF. Very few patients have paroxysmal $\mathrm{AF}$ triggered by foci within the pulmonary veins. Only in these later patients we could plan pulmonary vein isolation (PVI) as the only treatment for AF. In all other patients with persistent AF a strategy of a bipolar Maze should be considered, as suggested by the 2016 ESC/EACTS Guidelines (1). Bi-atrial Maze has shown clear better maintenance of sinus rhythm in these patients $(12,13)$. A recent randomized controlled trial in mitral patients suggested that PVI had the same effect than a full Maze lesion set, arising the controversy of PVI $v$ s. Maze in these patients, but Maze lines beyond PVI were performed with energies that are known to be no consistent to provoke linear, transmural lesions, which explains the poor results of both PVI and Maze in those patients (4).

\section{AF inducing mitral regurgitation?}

Conceptually, functional mitral regurgitation (type I of the Carpentier classification) needs mitral annulus dilatation, and the main cause for such dilatation is left ventricular dilatation with impaired systolic function (14). Usually, the dilated left ventricle may also compromise leaflet movement by distance augmentation from the annulus to the tip of the papillary muscle, provoking leaflets tenting during systole (type IIIb of the Carpentier classification). Recently, new evidence suggests that left atrial dilatation by AF may induce mitral regurgitation in patients with normal left ventricular size and global and segmental function, in what it is been called 'atrial functional mitral regurgitation' (15). Echo studies describe a normal mitral valve with a dilated left atrium, with an important mitral annulus dilatation and an important defect in leaflet coaptation length, similar to patients with ventricular dilatation and dysfunction. The main difference between the two groups is that leaflets in ventricular dysfunction are tethered by the subvalve apparatus and accordingly sit well below the level of the annulus. In patients with annular dilatation and regurgitation with $\mathrm{AF}$ and normal left ventricle, leaflets have unfolded and sit close to the annular plane (16). Although some authors describe that catheter ablation may correct $\mathrm{AF}$ and $\mathrm{AF}$-induced severe mitral regurgitation, we believe all patients with significant mitral insufficiency and AF should undergo cardiac surgery with Maze and mitral repair, as recommended by the 2016 ESC/EACTS Guidelines $(1,17)$.

\section{Technical details of AF ablation and mitral repair}

There are no special tricks to perform a Maze procedure concomitant to a mitral repair. The main objective is to achieve permanent, linear, transmural lines, with a standard pattern as described by Cox (18). Up to today, only cut-and-sew, bipolar radiofrequency and cryothermy can consistently perform permanent linear, transmural lines. Of special interest for this review is that the line to the mitral annulus must be consistent. Any small gap in this line may induce to flutter around the mitral annulus, usually at $120 \mathrm{bpm}$, very reluctant to medical treatment. Additionally, if the gap is underneath the mitral prosthetic ring, it is un-accessible by postoperative catheter ablation. This is why it is important to perform the full ablation before implanting a prosthetic ring (Figures 1,2).

\section{Conclusions}

AF is associated to mitral regurgitation. Since 2010, European Guidelines recommend surgery for mitral repair and ablation as soon as AF is detected even in asymptomatic patients, in order to avoid further atrial dysfunction and deterioration. Concomitant AF surgery has shown to be safe and effective in sinus rhythm maintenance compared to 


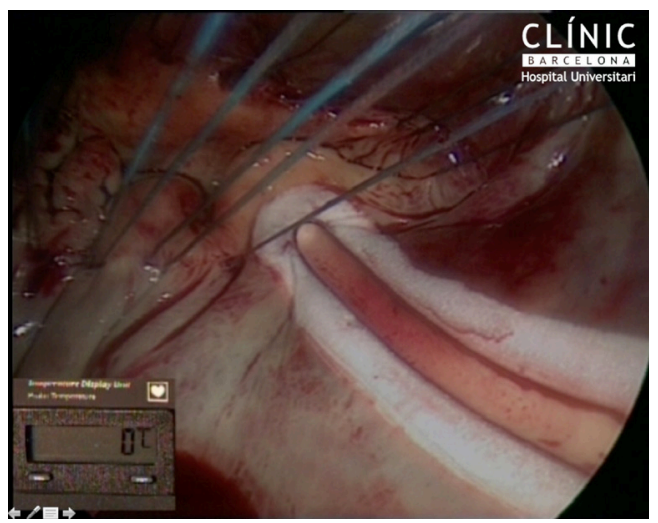

Figure 1 Cryothermy lesion performed after posterior leaflet triangular resection and placing sutures for the prosthetic ring, before implanting the ring.
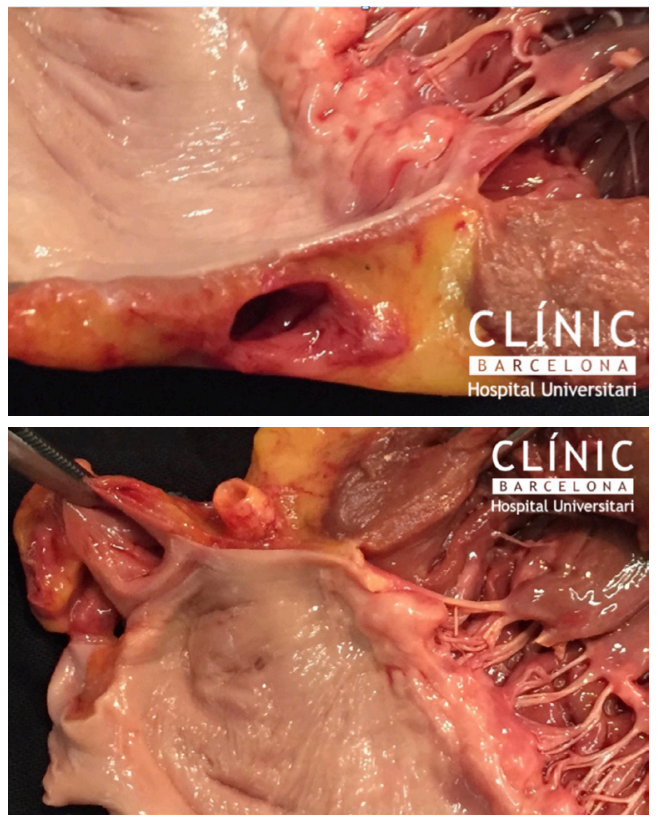

Figure 2 Note the close relationship between the atrial muscle, the mitral annulus, the coronary sinus and the circumflex artery. Ablation needs to be performed trying to avoid the circumflex artery. Since it is difficult to reach the mitral annulus with a radiofrequency clamp, an endocardial cryothermy application can finish the line to the annulus.

no ablation. Recent reports suggest that their benefits span to mortality and freedom from stroke. For better results, a full right and left Maze procedure should be performed in patients with mitral regurgitation and persistent $\mathrm{AF}$. In the rare cases in which mitral insufficiency is associated to paroxysmal AF, data suggest that PVI could be sufficient to maintain sinus rhythm.

There is few evidence suggesting left atrial dilatation due to AF may induce mitral regurgitation in patients with normal left ventricular function and size. This may happen in a small population of AF patients, and the main mechanism seems to be progressive annular dilatation that reduces the surface of coaptation between leaflets. While the discussion clarifies between who was first, the egg or the chicken, there is enough evidence to recommend that patients with mitral regurgitation and $\mathrm{AF}$ undergo mitral repair and ablation.

\section{Acknowledgements}

None.

\section{Footnote}

Conflicts of Interest: Dr. Castellá is a consultant for Atricure, Medtronic.

\section{References}

1. Kirchhof P, Benussi S, Kotecha D, et al. 2016 ESC Guidelines for the management of atrial fibrillation developed in collaboration with EACTS. Eur J Cardiothorac Surg 2016;50:e1-e88.

2. Lim E, Barlow CW, Hosseinpour AR, et al. Influence of atrial fibrillation on outcome following mitral valve repair. Circulation 2001;104:I59-63.

3. Huffman MD, Karmali KN, Berendsen MA, et al. Concomitant atrial fibrillation surgery for people undergoing cardiac surgery. Cochrane Database Syst Rev 2016;(8):CD011814.

4. Gillinov AM, Gelijns AC, Parides MK, et al. Surgical ablation of atrial fibrillation during mitral-valve surgery. $\mathrm{N}$ Engl J Med 2015;372:1399-409.

5. Bando K, Kobayashi J, Hirata M, et al. Early and late stroke after mitral valve replacement with a mechanical prosthesis: risk factor analysis of a 24-year experience. J Thorac Cardiovasc Surg 2003;126:358-64.

6. Bando K, Kobayashi J, Kosakai Y, et al. Impact of Cox maze procedure on outcome in patients with atrial fibrillation and mitral valve disease. J Thorac Cardiovasc Surg 2002;124:575-83.

7. Je HG, Lee JW, Jung SH, et al. Risk factors analysis on failure of maze procedure: mid-term results. Eur J 
Cardiothorac Surg 2009;36:272-8; discussion 378-9.

8. Louagie Y, Buche M, Eucher P, et al. Improved patient survival with concomitant Cox Maze III procedure compared with heart surgery alone. Ann Thorac Surg 2009;87:440-6.

9. Badhwar V, Rankin JS, Ad N, et al. Surgical Ablation of Atrial Fibrillation in the United States: Trends and Propensity Matched Outcomes. Ann Thorac Surg 2017;104:493-500.

10. Sunderland N, Maruthappu M, Nagendran M. What size of left atrium significantly impairs the success of maze surgery for atrial fibrillation? Interact Cardiovasc Thorac Surg 2011;13:332-8.

11. Ad N, Holmes SD. Prediction of sinus rhythm in patients undergoing concomitant Cox maze procedure through a median sternotomy. J Thorac Cardiovasc Surg 2014;148:881-6; discussion 886-7.

12. Gillinov AM, Bhavani S, Blackstone EH, et al. Surgery for permanent atrial fibrillation: impact of patient factors and lesion set. Ann Thorac Surg 2006;82:502-13; discussion 513-4.

13. Barnett SD, Ad N. Surgical ablation as treatment for the

doi: 10.21037 /jovs.2017.08.22

Cite this article as: Castellá $M$. Atrial fibrillation surgery and mitral repair. J Vis Surg 2017. elimination of atrial fibrillation: a meta-analysis. J Thorac Cardiovasc Surg 2006;131:1029-35.

14. Otsuji Y, Kumanohoso T, Yoshifuku S, et al. Isolated annular dilation does not usually cause important functional mitral regurgitation: comparison between patients with lone atrial fibrillation and those with idiopathic or ischemic cardiomyopathy. J Am Coll Cardiol 2002;39:1651-6.

15. Kihara T, Gillinov AM, Takasaki K, et al. Mitral regurgitation associated with mitral annular dilation in patients with lone atrial fibrillation: an echocardiographic study. Echocardiography 2009;26:885-9.

16. Ring L, Dutka DP, Wells FC, et al. Mechanisms of atrial mitral regurgitation: insights using $3 \mathrm{D}$ transoesophageal echo. Eur Heart J Cardiovasc Imaging 2014;15:500-8.

17. Gertz ZM, Raina A, Saghy L, et al. Evidence of atrial functional mitral regurgitation due to atrial fibrillation: reversal with arrhythmia control. J Am Coll Cardiol 2011;58:1474-81.

18. Cox JL. The surgical treatment of atrial fibrillation. IV. Surgical technique. J Thorac Cardiovasc Surg 1991;101:584-92. 